\title{
Estimation of the degree of gastric mucosal atrophy based on serum pepsinogen levels after eradication of Helicobacter pylori
}

Takahiro Suzuki

Hamamatsu Ika Daigaku

Tomohiro Higuchi

Hamamatsu Ika Daigaku

Takuma Kagami

Hamamatsu Ika Daigaku

Mihoko Yamade

Hamamatsu Ika Daigaku

Shinya Tani

Hamamatsu Ika Daigaku

Yasushi Hamaya

Hamamatsu Ika Daigaku

Moriya Iwaizumi

Hamamatsu Ika Daigaku

Satoshi Osawa

Hamamatsu Ika Daigaku

Ken Sugimoto

Hamamatsu Ika Daigaku

Hiroaki Miyajima

Hamamatsu Ika Daigaku

Takahisa Furuta ( $\nabla$ furuta@hama-med.ac.jp)

Hamamatsu University School of Medicine https://orcid.org/0000-0002-2202-595X

Research article

Keywords: Atrophic gastritis, eradication, Helicobacter pylori, Pepsinogen

Posted Date: August 13th, 2020

DOI: https://doi.org/10.21203/rs.3.rs-42652/v1 
License: (c) (i) This work is licensed under a Creative Commons Attribution 4.0 International License. Read Full License 


\section{Abstract \\ Background}

Serum pepsinogen (PG) levels correlate with the degree of gastric mucosal inflammation and atrophy, which correlate with gastric cancer risk, in patients infected with Helicobacter pylori $(H$. pylori). Serum PG levels change after eradication of $H$. pylori, but it is not known if there are corresponding changes in the gastric mucosa. We examined whether the degree of gastric atrophy correlated with PG levels measured after eradication of $H$. pylori.

\section{Methods}

We retrospectively examined the relationship between gastric atrophy and serum levels of PG I, PG II and PG I/II ratios measured after eradication of $H$. pylori. The degree of gastric mucosal atrophy before $H$. pylori eradication was scored $(0,1,2)$ according to the Kyoto classification of gastritis.

\section{Results}

A total of 430 treated patients were enrolled. Serum levels of PG I $(\rho=-0.362$ and P< 0.001$)$, PG II $(\rho=-$ 0.158 and $P=0.001)$ and $P G I / I I$ ratio $(\rho=-0.337$ and $P<0.001)$ all correlated negatively with atrophy scores. When PG I/II was less than $3.4,5.4$ or 6.7 , the probability of the open type of gastric atrophy was estimated to be $75 \%, 50 \%$, or $25 \%$, respectively.

\section{Conclusion}

Our results suggest that serum PG levels measured after $H$. pylori eradication can be used to estimate the degree of gastric mucosal atrophy and are useful for selecting individuals with a high risk of gastric cancer after $H$. pylori eradication.

\section{Background}

Gastric cancer (GC) is the third-most common cause of cancer-related mortality worldwide ${ }^{1}$. Helicobacter pylori (H. pylori) is one of the main causes of $\mathrm{GC}^{2}$. The Kyoto global consensus report on H. pylori gastritis recommends that all individuals with $H$. pylori infection receive eradication therapy to prevent $\mathrm{GC}^{3,4}$. In Japan, health insurance has covered eradication therapy in all patients infected with $H$. pylori since 2013, and infection have been cleared in many patients. Although the risk of GC is decreased by $H$. pylori eradication ${ }^{5-8}$, gastric cancer risk nevertheless remains high in subjects with severe gastric atrophy ${ }^{9}$. Therefore, a biomarker for severe gastric atrophy that can be used after eradication of $H$. pylori would be useful. 
Endoscopic screening is useful not only to detect GC in the early stage but also to evaluate the GC risk based on findings in the gastric mucosa. Annual endoscopic screening is expected to reduce the number of deaths due to $\mathrm{GC}^{10}$. Although the Japanese government has decided to introduce endoscopic $\mathrm{GC}$ screening nationwide, not all candidates for examination undergo screening. Some patients hesitate to undergo endoscopy because of its invasiveness. Moreover, some patients may be precluded from endoscopy due to insufficient medical resources. A noninvasive cost-effective tool that selects subjects at higher risk of severe gastric atrophy would therefore make screening more attractive and efficient.

Serum pepsinogen (PG) levels are well known to reflect the severity of gastric mucosal atrophy and inflammation in patients infected with H. pylori. Low serum PG I levels and low PG I/II ratios can predict the grade of gastric mucosal atrophy and individual risk of GC development ${ }^{11}$, and are used to select the patients at the highest risk of gastric cancer for combination assay for anti-H. pylori lgG antibody ${ }^{12}$.

PGI and PG II are known to decrease, and PG I/II ratio to increase, as gastric mucosal inflammation improves post-treatment ${ }^{13}$. To evaluate gastric cancer risk, serum levels of PGs should ideally be measured before eradication of $H$. pylori, but most patients undergo eradication therapy without pretreatment measurement of serum levels of PGs. It is difficult to apply the original diagnostic criteria of serum PGs to subjects with successful eradication of $H$. pylori because the relationship among posttreatment serum PGs, gastric atrophy, and gastric cancer risk has not been fully studied.

The Kyoto classification of gastritis ${ }^{14}$ was established in 2015 to characterize gastric mucosa infected with $H$. pylori, gastric mucosa after eradication of $H$. pylori, and gastric mucosa never infected with $H$. pylori. This classification is also useful for the evaluation of gastric cancer risk ${ }^{15}$. Of several parameters used to describe the type and grade of gastritis, atrophy is one of the most important parameter linked to gastric cancer risk. However, the relationship between the Kyoto classification of gastritis and serum PGs has not been clarified.

Here, to examine whether serum PG levels are useful for estimating the degree of residual gastric mucosal atrophy, we examined the relationship between posttreatment serum PG levels and gastric mucosal atrophy using the Kyoto classification ${ }^{14}$.

\section{Methods}

\section{Study design and oversight}

The study was conducted under a retrospective design. The primary end point was to evaluate the relationship between the degree of gastric atrophy found by endoscopy and posttreatment serum levels of PG I and PG II and the PG I/II ratio. The secondary end point included whether serum PGs measured after eradication of $H$. pylori can be used to predict the grade of gastric atrophy.

The protocol was in advance approved by the ethics committee of Hamamatsu University School of Medicine (R19-188). 
The first draft of the manuscript was written by the first author with corresponding author. Then, English medical writers (G.H., L.C.) revised the manuscript. Before submission, all authors reviewed and revised the manuscript. The decision of the submission of the manuscript for publication was made by all authors.

\section{Subjects}

The cases of 1207 patients who had visited the H. pylori-specific outpatient unit of the hospital of Hamamatsu University School of Medicine (1-20-1, Handayama, Higashi-ku, Hamamatsu, 431-3192, Japan) from August 2010 to December 2018 were screened from the medical records. Written informed consent for the use of their treatment results for medical research was obtained from each of all patients at the first visit to the hospital.

Patients were included if they had undergone esophagogastroduodenoscopy (EGD) before H. pylori eradication and measurement of serum levels of PG I and II after $H$. pylori eradication, and excluded if they had total or partial gastrectomy, $H$. pylori eradication failure, or had never been infected with $H$. pylori.

Patients underwent EGD, which included careful investigation of the esophagus, stomach, and duodenum and of the status of $H$. pylori infection-associated gastric atrophy. H. pylori infection was confirmed by a rapid urease test (RUT) (Helico Check; Otsuka Pharmaceutical Co. Ltd., Tokyo, Japan). Gastric atrophy was divided into closed or open types based on the Kyoto classification of gastritis. An atrophy score of 0 (A0) implies atrophy limited to the antrum ( $\mathrm{CO}-\mathrm{Cl}$ according to the Kimura-Takemoto classification $\left.{ }^{16}\right)$. An atrophy score of 1 (A1) implies that the atrophic area was limited to the body but did not include the cardia (C-II-C-III according to the Kimura-Takemoto classification). An atrophy score of 2 (A2) means atrophy area extending from the antrum to over the cardia (OI-OIII according to Kimura-Takemoto classification). A0 plus A1 atrophy corresponds to the closed type of atrophic gastritis and A2 corresponds to the open type of atrophic gastritis, based on the Kimura-Takemoto classification.

Patients infected with $H$. pylori underwent eradication therapy. One to two months after therapy, they underwent another $H$. pylori $\left[{ }^{13} \mathrm{C}\right]$-urea breath test (UBT) to assess whether eradication was successful.

\section{Serum pepsinogen}

Blood samples were collected from subjects in the morning 6-24 months after $H$. pylori eradication. Serum levels of PG I and PG II (LZ-PEPSINOGEN I and II, Eiken Chemical Co., Ltd., Tokyo, Japan) were measured. All blood samples were centrifuged immediately after collection at $3000 \mathrm{rpm}$. Serum samples were stored at $-20^{\circ} \mathrm{C}$ until analysis. All measurements were performed at a commercial laboratory (SRL, Inc., Kanagawa, Japan).

\section{Statistical analysis}


Whether medians of PG I, PG II and PG I/II ratio correlated with the atrophy scores were assessed by Spearman's rank correlation coefficient. The relationship between the atrophy score and PGs was determined using the Kruskal Wallis test followed by the Mann-Whitney U-test. We analyzed the relationship of serum PG I/II ratio and the probability of open or closed types of gastric atrophy using Kaplan Meier curves. Statistical calculations were performed with commercial software (SPSS version 25; IBM SPSS, Chicago IL, USA). All P values were two-tailed, and $\mathrm{P}<0.05$ indicated statistical significance.

\section{Results}

\section{Clinical characteristics of subjects}

A total of 1207 cases were screened. Of them, 430 cases whose serum levels of PG I and II were measured after $\mathrm{H}$. pylori eradication and whose endoscopic findings of gastric mucosa were recorded before $H$. pylori eradication were selected for the analyses (Figure 1). Clinical characteristics of these cases are summarized in Table 1.

\section{Comparison of median serum PG levels}

Median (with range) serum PG I levels $(\mathrm{ng} / \mathrm{mL}$ ) measured after eradication of $H$. pylori in patients with atrophy scores of 0,1 , and 2 of atrophy scores were 44.1 (26.8-96.7), 40.6 (7.3-368.0) and 28.4 (2.0225.0), respectively (Figure 2a). Serum PG II levels were 8.4 (6.2-16.7), 7.9 (2.0-63.0) and 6.9 (2.4-36.9), respectively (Figure $2 b)$. PG I/II ratios were $4.9(4.0-8.1), 5.2(1.1-9.1)$ and $4.0(0.4-11.0)$, respectively (Figure 2c). Results showed a negative correlation: the higher the atrophy score, the lower the PG I, PG II, and $P G \mathrm{I} / \mathrm{II}$ values.

Serum levels of PG I, PG II and PG I/II ratio levels measured after eradication of $H$. pylori were all negatively correlated with atrophy score (PG I: $r=-0.362, P<0.001 ; P G ~ I I: r=-0.158, P=0.001 ; P G ~ I / P G ~ I I$ ratio: $r=-0.337, P<0.001)$.

We analyzed these data using Kaplan Meier curves to predict the values for open type atrophy (Figure 3a, $3 b$ and $3 c)$.

ROC Curves are shown in Figure 4. Of the three parameters, the $P G \mathrm{I} / \mathrm{Il}$ ratio was the best to estimate the risk for open atrophy. When the posttreatment serum PG I/II ratio measured $<3.4$, the probability of open type atrophy was $>75 \%$ (Figure 5 and Table 2 ). When serum PG I/II ratio was approximately 5.4 , the probability of open type atrophy was approximately $50 \%$. When serum PG I/II ratio was $>6.7$, the probability of open type atrophy was $<25 \%$. Similar phenomenon was observed for PG I and PG II. Accordingly, serum PGs could be used to predict the probability of the grade of gastric mucosal atrophy.

\section{Discussion}


We found that serum PG levels measured after eradication of $H$. pylori were useful in predicting the degree of gastric mucosal atrophy. We demonstrated that serum levels of PG I, PG II and PG I/II ratio measured after eradication of $H$. pylori each differed between the groups with close and open types of gastric atrophy, that as gastric atrophy progressed, they were decreased, as observed in serum PG levels measured before eradication of $H$. pylori and that the PG I/II ratio was most useful of the three markers in estimating the possibility of open gastric atrophy.

The serum levels of PG I and PG II both decreased posttreatment ${ }^{17}$. As the degree of decrease of PG II levels was greater than that of PG I, the PG I/II ratio increased after treatment ${ }^{18}$. After H. pylori eradication, the mucosal inflammation improved, then the influence of inflammation on serum PG levels would be decreased. Therefore, serum PG levels measured after eradication of $H$. pylori are considered to purely express the degree of the gastric mucosal atrophy. Because the open type of gastric atrophy is associated with a higher risk of gastric cancer than the closed type ${ }^{2}$, serum PG levels measured after treatment may also be useful in selecting patients at an elevated risk of gastric cancer.

Haneda et al. ${ }^{19}$ reported that a serum PG I/II ratio $\leq 4.5$ is a useful cutoff to select those at risk for gastric cancer after eradication of $H$. pylori. The sensitivity of this cutoff value for the development of gastric cancer after eradication of $H$. pylori was reported to be $65.9 \%$. In their study, sensitivity in identifying gastric cancer by the PG method was 48.9\%. Therefore, serum PGs measured after eradication of $H$. pylori were considered as useful as those measured before eradication. The number of studies on the usefulness of serum PGs measured after treatment is still very low, and further studies are needed.

In this study, we demonstrated that the probability of open gastric atrophy could be estimated by serum PG I/II ratio measured after eradication of $H$. pylori. Usually, the optimal cutoff value is set to select the subjects at highest gastric cancer risk ${ }^{19-21}$. However, there are some patients who could not be selected based on the cutoff value. By indicating the individual risk for severe gastric atrophy by percentage, each patient may better understand the condition of his or her own stomach, and be more amenable to endoscopic follow up.

In Japan, endoscopic examination for gastric cancer is widespread, but resources are limited, indicating the need for risk stratification. The evaluation of gastric cancer risk based on the endoscopic findings of gastric mucosa is useful. Endoscopy is operator-dependent. Gastrography is also useful in evaluating gastric atrophy. However, gastrography is thought to be inferior to endoscopy with regard to the detection rate of gastric cancer in the early stage. Serum PGs before eradication of $H$. pylori have been considered useful in evaluating gastric cancer risk objectively. In the present study, serum PGs measured after eradication of $\mathrm{H}$. pylori were demonstrated to be useful in estimating gastric atrophy, and may contribute to the selection of candidates for gastric cancer screening by endoscopy.

This study had some notable limitations. First, the observation period was 6-24 months. It is unknown whether PG levels measured a substantial period after eradication of $H$. pylori are accurate predictors of risk. Second, we measured serum PG levels in the morning only, although there is circadian variation in 
serum pepsinogen levels. Third, our subjects were all Japanese. Fourth, the study was conducted under a single-center retrospective design. Finally, our study results were obtained from the training set data and must be verified by the validation set. Given these limitations, our study should be considered preliminary, and further prospective multicenter studies are needed to clarify the utility of serum pepsinogens measurement after eradication of H. pylori.

\section{Conclusion}

We reported the utility of a gastric atrophy risk stratification system using serum PGs measured after eradication of $H$. pylori. The serum PG I/II ratio was the most useful marker for the estimation of gastric atrophy. Although it is desirable to examine PG levels before eradication, objective evaluation of gastric cancer risk after eradication of $\mathrm{H}$. pylori is possible using serum PGs, and may be of clinical importance if it encourages at-risk individuals to participate in gastric cancer screening. We expect that further clinical studies will be performed to demonstrate the clinical importance of serum PGs measurement after eradication of $\mathrm{H}$. pylori, and that measurement will be incorporated into daily clinical practice.

\section{Abbreviations}

GC: Gastric cancer, H. pylori: Helicobacter pylori, PG: pepsinogen,

\section{Declarations}

- Ethics approval and consent to participate

The protocol was approved by the ethics committee of Hamamatsu University School of Medicine (R19188). The consent to participate was opt-out.

- Consent for publication

All authors agreed with the submission of the manuscript for publication.

- Availability of data and material

Not applicable

- Competing interests

The authors declare that there is no competing interest regarding the publication of this article.

- Funding

This study was performed by the self funding.

- Authors' contributions 
TS: Plan of the study, patient care, endoscopy of patients, statistical analysis and interpretation of the data and drafting of the manuscript.

TH: interpretation of the data, endoscopy of patients and checking the manuscript.

TK: Plan of the study, endoscopy of patients and checking the manuscript.

MY: Plan of the study, patient care, endoscopy of patients and checking the manuscript.

ST: Patient care, endoscopy of patients and checking the manuscript.

YH: Endoscopy of patients

Ml: Patient care

SO: Patient care, endoscopy of patients and checking the manuscript.

HM: Patient care and checking the manuscript.

KS: Patient care, endoscopy of patients and checking the manuscript.

TF: Plan of the study, patient care, endoscopy of patients, interpretation of the data and revision of the manuscript.

All authors have read and approved the manuscript

- Acknowledgements

We greatly appreciate the help of the staff of the endoscopy unit, namely Ms. Hiroko Iwata, Ms. Yoko Suzuki, Ms. Junko Ishiduka, Ms. Azusa Umeda, Ms. Naoko Kamiya, Ms. Rieko Kawai, Ms. Noriko Kawakami, Ms. Chiaki Kawaguchi, Ms. Tomoko Kumagai, Ms. Yumie Suganuma, Ms. Mari Suzuki, Ms. Nobue Takahashi, Ms. Naoko Fujimoto, Ms. Chieko Matsumoto, Ms. Hisako Murase, Ms. Natsumi Ikuma, Ms. Keiko Kikuchi, Ms. Chikako Sasagase, Ms. Mieko Suzuki, Ms. Satoko Takebayashi, Ms. Emiko Tomatsu, Ms. Kinuko Maruyama and Ms. Atsumi Murai.

\section{References}

1. World Health Organization. Available online: http://gco.iarc.fr/today/home (accessed on 3 July 2019).

2. Uemura N, Okamoto S, Yamamoto S, et al. Helicobacter pylori infection and the development of gastric cancer. The New England journal of Medicine 2001;345:784-9.

3. Sugano K, Tack J, Kuipers EJ, et al. Kyoto global consensus report on Helicobacter pylori gastritis. Gut 2015;64:1353-67. 
4. Suzuki H, Mori H. Helicobacter pylori: Helicobacter pylori gastritis-a novel distinct disease entity. Nature reviews Gastroenterology \& hepatology 2015;12:556-7.

5. Kato M, Ota H, Okuda M, et al. Guidelines for the management of Helicobacter pylori infection in Japan: 2016 Revised Edition. Helicobacter 2019:e12597.

6. Ford AC, Forman D, Hunt RH, Yuan Y, Moayyedi P. Helicobacter pylori eradication therapy to prevent gastric cancer in healthy asymptomatic infected individuals: systematic review and meta-analysis of randomised controlled trials. BMJ (Clinical research ed) 2014;348:g3174.

7. Zhao B, Zhang J, Mei D, et al. Does Helicobacter pylori Eradication Reduce the Incidence of Metachronous Gastric Cancer After Curative Endoscopic Resection of Early Gastric Cancer: A Systematic Review and Meta-Analysis. Journal of clinical gastroenterology 2019.

8. Sugano K. Effect of Helicobacter pylori eradication on the incidence of gastric cancer: a systematic review and meta-analysis. Gastric cancer : official journal of the International Gastric Cancer Association and the Japanese Gastric Cancer Association 2019;22:435-45.

9. Take S, Mizuno M, Ishiki K, et al. The long-term risk of gastric cancer after the successful eradication of Helicobacter pylori. Journal of gastroenterology 2011;46:318-24.

10. Zhang X, Li M, Chen S, et al. Endoscopic Screening in Asian Countries Is Associated With Reduced Gastric Cancer Mortality: A Meta-analysis and Systematic Review. Gastroenterology 2018;155:34754.e9.

11. Kiyohira K, Yoshihara M, Ito M, Haruma K, Tanaka S, Chayama K. Serum pepsinogen concentration as a marker of Helicobacter pyloriinfection and the histologic grade of gastritis; evaluation of gastric mucosa by serum pepsinogen levels. Journal of gastroenterology 2003;38:332-8.

12. Osumi $\mathrm{H}$, Fujisaki $\mathrm{J}$, Suganuma $\mathrm{T}$, et al. A significant increase in the pepsinogen $\mathrm{I} / \mathrm{II}$ ratio is a reliable biomarker for successful Helicobacter pylori eradication. PloS one 2017;12:e0183980.

13. Furuta T, Kaneko E, Baba S, Arai H, Futami H. Percentage changes in serum pepsinogens are useful as indices of eradication of Helicobacter pylori. The American journal of gastroenterology 1997;92:84-8.

14. Kamada T, Haruma K, Inoue K, Shiotani A. [Helicobacter pylori infection and endoscopic gastritis Kyoto classification of gastritis]. Nihon Shokakibyo Gakkai zasshi = The Japanese journal of gastroenterology 2015;112:982-93.

15. Sugimoto M, Ban $\mathrm{H}$, Ichikawa $\mathrm{H}$, et al. Efficacy of the Kyoto Classification of Gastritis in Identifying Patients at High Risk for Gastric Cancer. Internal medicine (Tokyo, Japan) 2017;56:579-86.

16. Kimura K, Takemoto T. An endoscopic recognition of the atrophic border and its significance in chronic gastritis. Endoscopy 1969;1:87-97.

17. Ohkusa $T$, Miwa $H$, Nomura $T$, et al. Improvement in serum pepsinogens and gastrin in long-term monitoring after eradication of Helicobacter pylori: comparison with $\mathrm{H}$. pylori-negative patients. Alimentary pharmacology \& therapeutics 2004;20 Suppl 1:25-32.

18. Massarrat S, Haj-Sheykholeslami A, Mohamadkhani A, et al. Pepsinogen II can be a potential surrogate marker of morphological changes in corpus before and after $\mathrm{H}$. pylori eradication. BioMed 
research international 2014;2014:481607.

19. Haneda $M$, Kato $M$, Ishigaki $S$, et al. Identification of a high risk gastric cancer group using serum pepsinogen after successful eradication of Helicobacter pylori. Journal of gastroenterology and hepatology 2013;28:78-83.

20. Chinda D, Shimoyama T, Mikami T, et al. Serum pepsinogen levels indicate the requirement of upper gastrointestinal endoscopy among Group A subjects of ABC classification: a multicenter study. Journal of gastroenterology 2018;53:924-31.

21. Nasrollahzadeh D, Aghcheli K, Sotoudeh $M$, et al. Accuracy and cut-off values of pepsinogens I, II and gastrin 17 for diagnosis of gastric fundic atrophy: influence of gastritis. PloS one 2011;6:e26957.

\section{Tables}

Table 1. Demographic and clinical characteristics of 430 cases.

\begin{tabular}{|ll|}
\hline Sex $(\mathrm{F} / \mathrm{M})$ & $205: 225$ \\
\hline Age, $\mathrm{y}$ (median with range) & $61(17-87)$ \\
\hline $\mathrm{PG} \mathrm{I}, \mathrm{ng} / \mathrm{mL}$ (median with range) & $33.8(2.0-368.0)$ \\
\hline $\mathrm{PG} I \mathrm{I}, \mathrm{ng} / \mathrm{mL}$ (median with range) & $7.3(2.0-63.0)$ \\
\hline $\mathrm{PG} \mathrm{I} / \mathrm{II}$ ratio (median with range) & $4.6(0.4-11.0)$ \\
\hline Anti- $H p$ IgG antibody, $\mathrm{U} / \mathrm{ml}$ (median with range) & $23.0(0.3-173.0)$ \\
\hline Gastric atrophy grade $(0,1,2)$ & $17: 155: 258$ \\
\hline
\end{tabular}

Table 2. The predicted probability of the grade of gastric mucosal atrophy.

\begin{tabular}{|llll|}
\hline Atrophy & \multicolumn{3}{l|}{ Closed to open } \\
\hline & $25 \%$ & $50 \%$ & $75 \%$ \\
\hline PG I (ng/mL) & 69.8 & 41.0 & 22.9 \\
\hline PG II (ng/mL) & 11.0 & 8.1 & 6.3 \\
\hline PG I/II ratio & 6.7 & 5.4 & 3.4 \\
\hline
\end{tabular}

\section{Figures}


Figure 1.

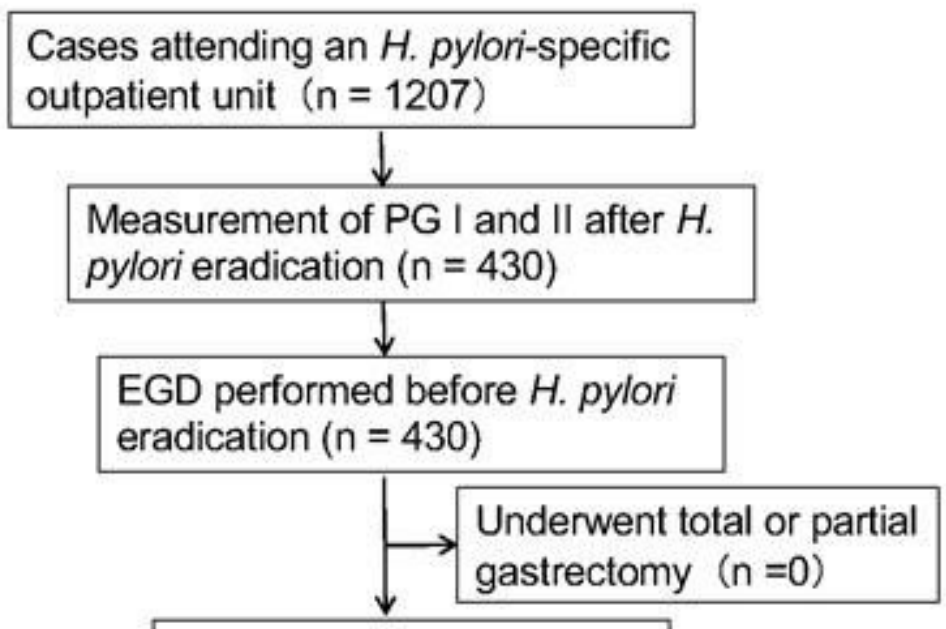

Finally enrolled $(n=430)$

\section{Figure 1}

Flow of enrollment of patients into the study.

Figure 2a.

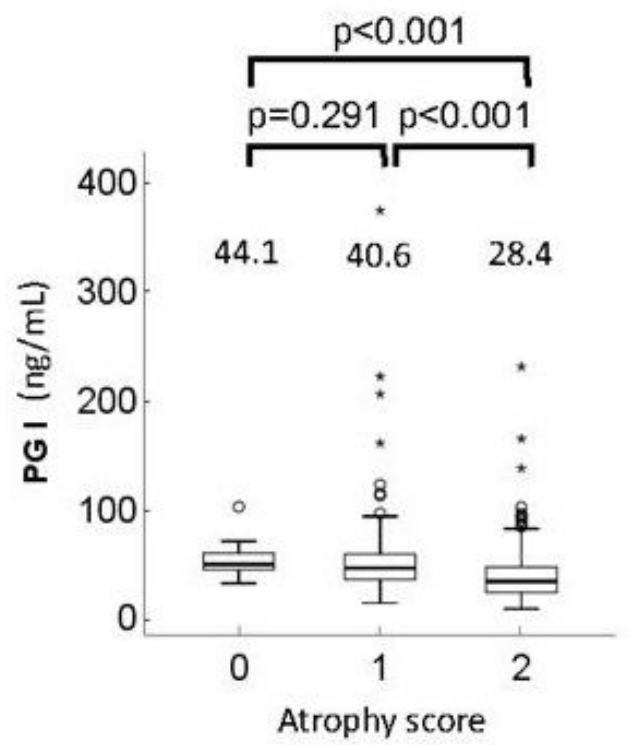

Figure 2b.

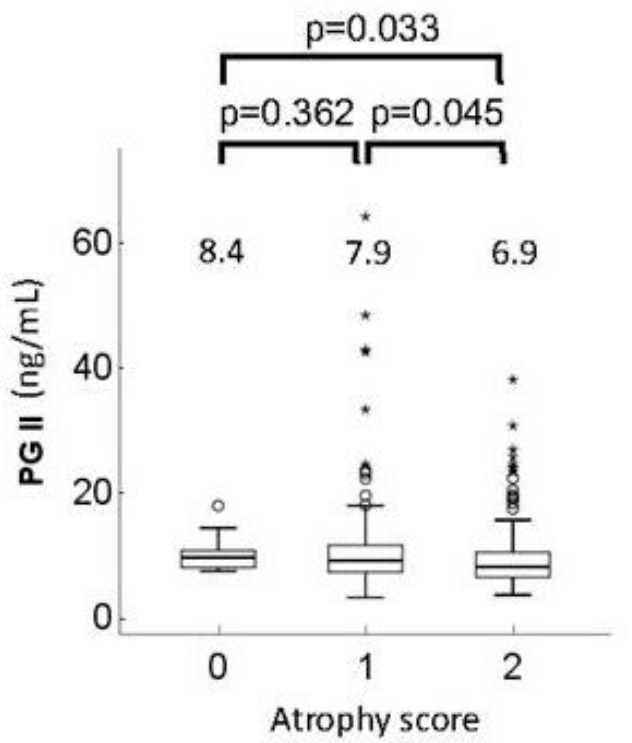

Figure 2c.

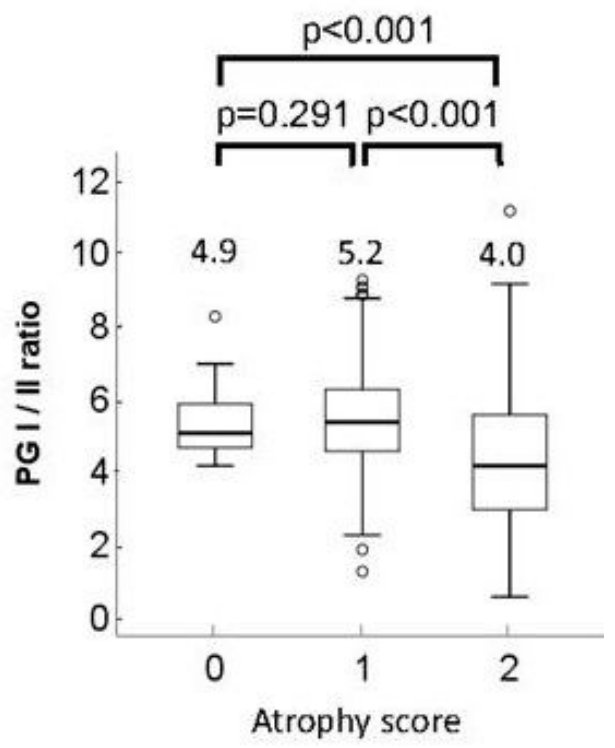

Figure 2 
Relationship between atrophy score $(0,1,2)$ and serum levels of PG I (a) and PG II (b), and PG I/II ratio (c).

Figure 3a.

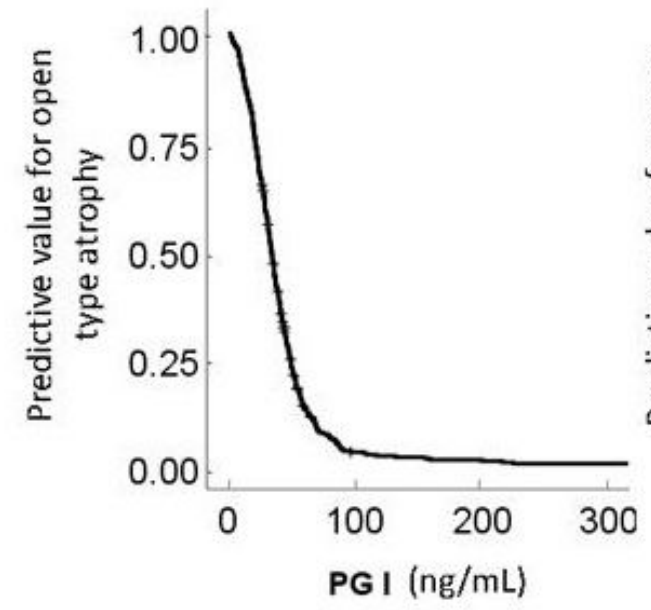

Figure $3 b$.

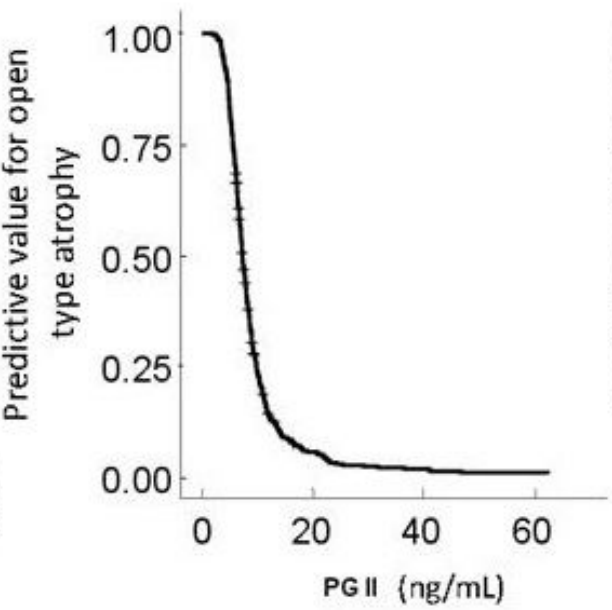

Figure 3c.

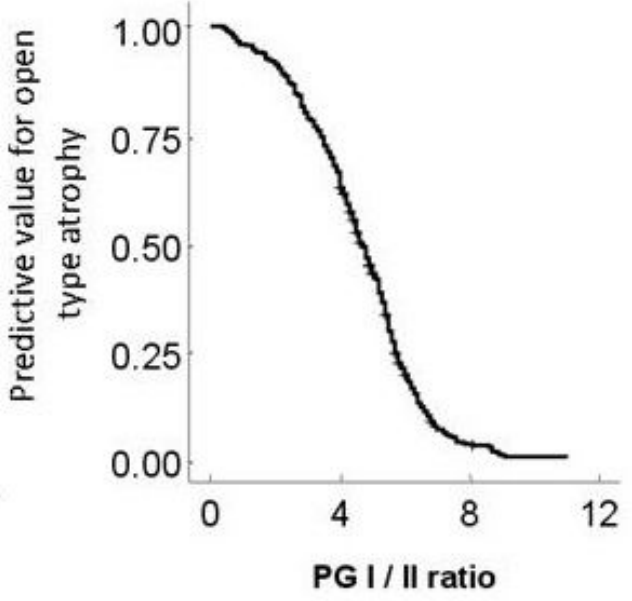

\section{Figure 3}

Relationship between serum PGs (a: PG I, b: PG II, c: PG I/II ratio) and predictive value for open gastric atrophy.

Figure 4.

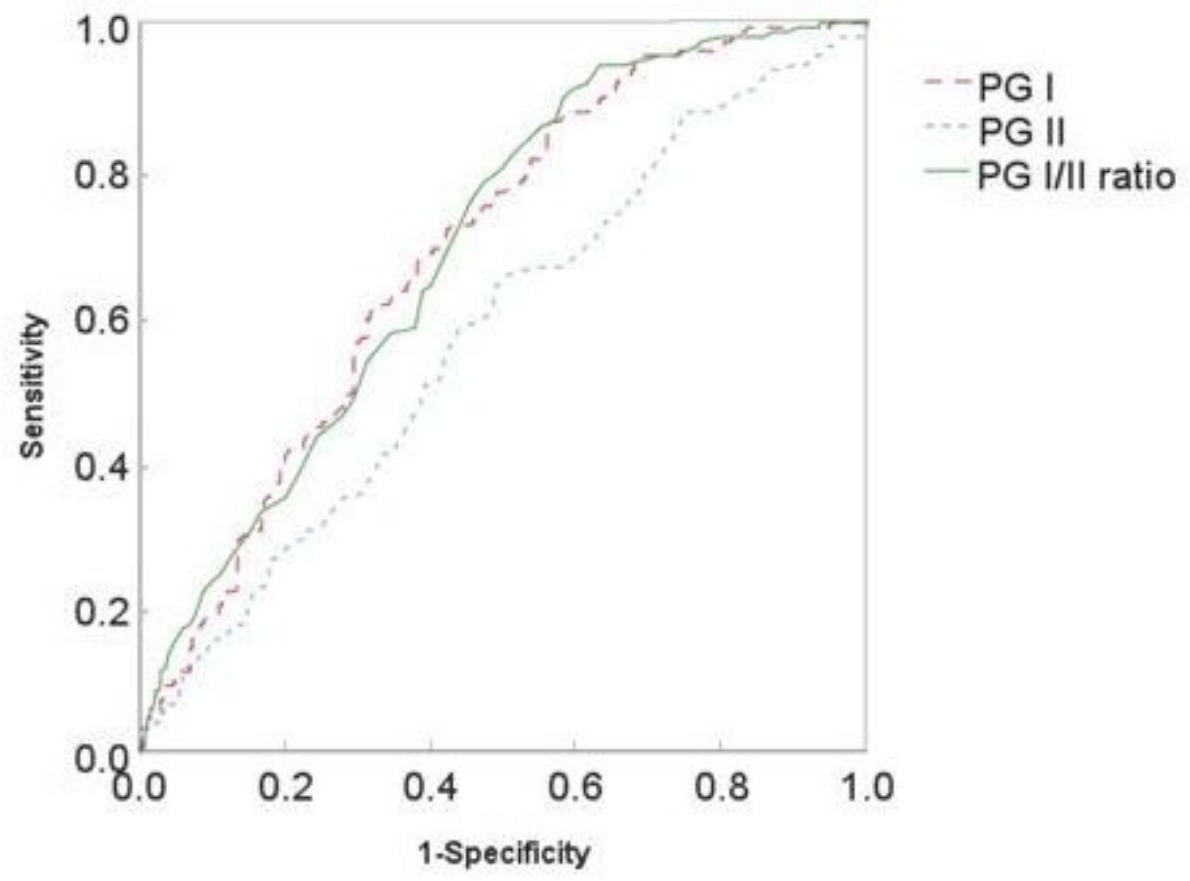


Figure 4

ROC analyses of PG I, PG II and PG I/II ratio as markers to distinguish open gastric atrophy from the closed type.

Figure 5 .

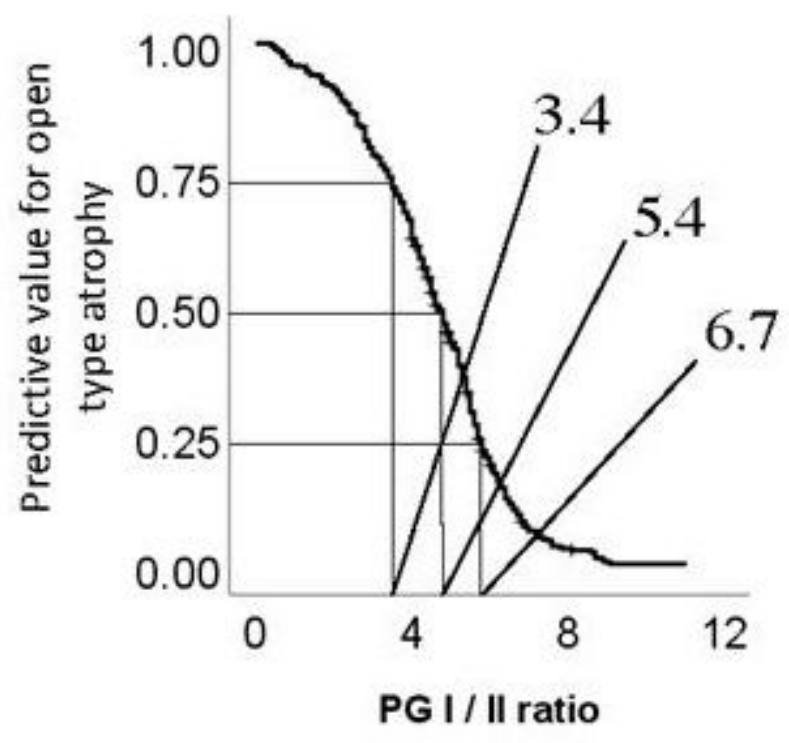

Figure 5

Estimation of the probability of gastric atrophy by serum PG I/II ratio measured after eradication of $\mathrm{H}$. pylori. 